\title{
Determination of projected potential profiles across interfaces using through focal series reconstruction
}

Somnath Bhattacharyya, Christoph T. Koch, and Manfred Rühle

Max-Planck-Institut für Metallforschung, Heisenberg Str 3, 70569, Stuttgart Germany

Potential profiles across interfaces can provide useful information about variations in structure, composition, density, ionicity of their constituents, the presence of space charge layers, etc, showing that the ability to measure potential profiles across interfaces is of great importance in the field of material science.

Transmission electron microscopy (TEM) techniques, based on phase contrast imaging, can determine local variations in electrostatic potential. In this work we report on a new, parameter-free a method to determine local variations in the mean inner potential from a series of Fresnel contrast image line profiles across an interface. A modified version of the iterative wave function reconstruction (IWFR) [1], is applied to reconstruct the exit face wave function from a through focal series of TEM image line profiles across an interface. Apart from high-resolution images recorded with small changes in defocus this method works also well for a large defocus range as used for Fresnel imaging. Using the phase-object approximation the projected electrostatic (elastic interaction of the electrons with the specimen) as well as the absorptive potential (inelastic scattering in addition with elastic scattering outside the objective aperture and thermal diffused scattering) profiles across an interface are determined from this exit face wave function. A new alignment procedure of experimental line profiles has been introduced and its robustness in the presence of high noise and uncertainties in defocus has been tested using simulated images. For the present study, it produces the best alignment in comparison with alignment procedures existing in the literature [2]. The reconstruction method is applied to both simulated and experimental images. For simulation it is shown to retrieve a potential drop across the interface quite accurately. For experimental data, mean inner potentials retrieved by this method agreed well with literature values [3], as shown in Fig. 1. Fig. 2 shows that while the reconstructed absorptive potential depends strongly on the local diffraction conditions and thus dynamical scattering, the relative reconstructed phase does not. It proves the validity of the phase object approximation for thicker specimen also. The resolution of the reconstruction is determined by the size of the objective aperture, as was confirmed by tests on an abrupt interface.

\section{References}

[1] L.J. Allen et al. Ultramicroscopy 100 (2004) 91.

[2] R.R. Meyer, A.I. Kirkland, W.O. Saxton, Ultramicroscopy 92 (2002) 89.

[3] C.T. Koch et al. Microsc. Microanal., in press.

[4] The financial assistance from the European Commission under contract Nr.s G5RD-CT-2001-00586 and NMP3-CT-2005-013862. Heartfelt thanks are expressed for Mr. Kersten Hahn, Dr. Gunther Richter, Ms. Limei Cha Dr. Raphaelle Satet and Prof. Michael Hoffmann. 

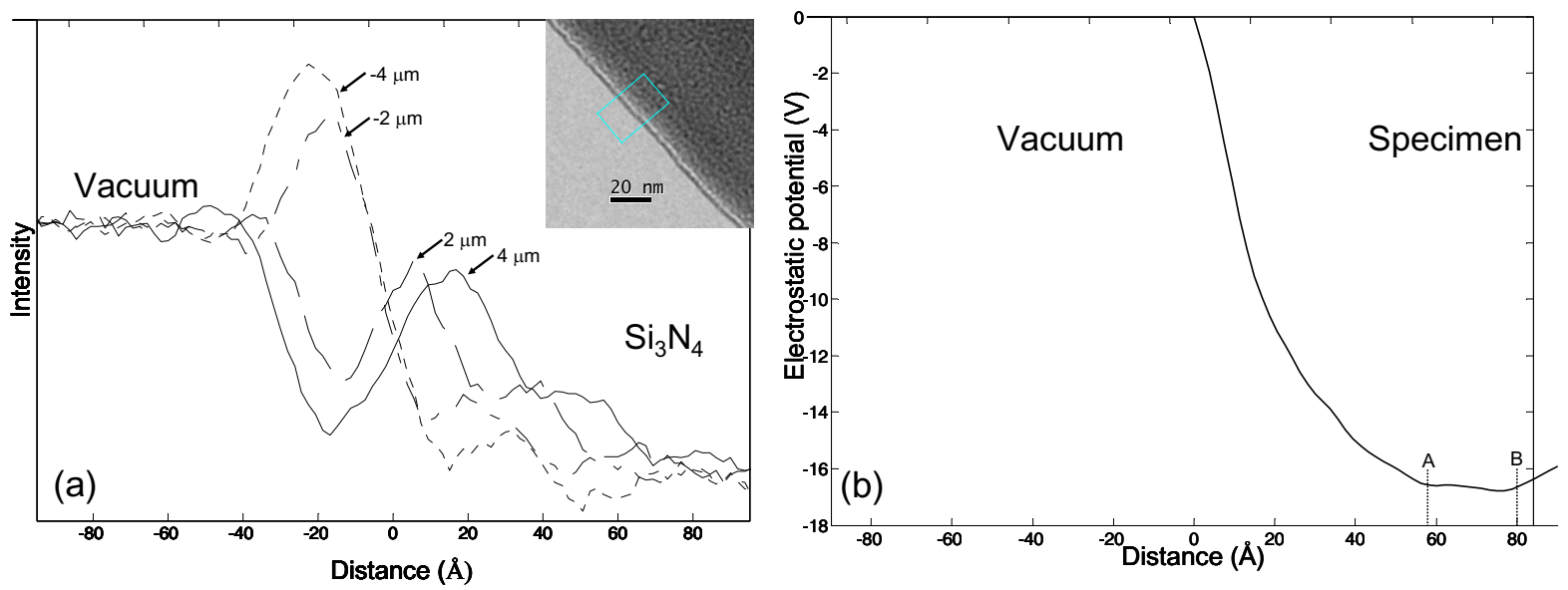

Fig. 1.(a) Four experimental 1D intensity profiles across this interface (corresponding defocus values are indicated in the figure). The inset shows an image of the vacuum$\mathrm{Si}_{3} \mathrm{~N}_{4}$ interface at an overfocus value of $1.1 \mu \mathrm{m}$. (b) reconstructed electrostatic potential.
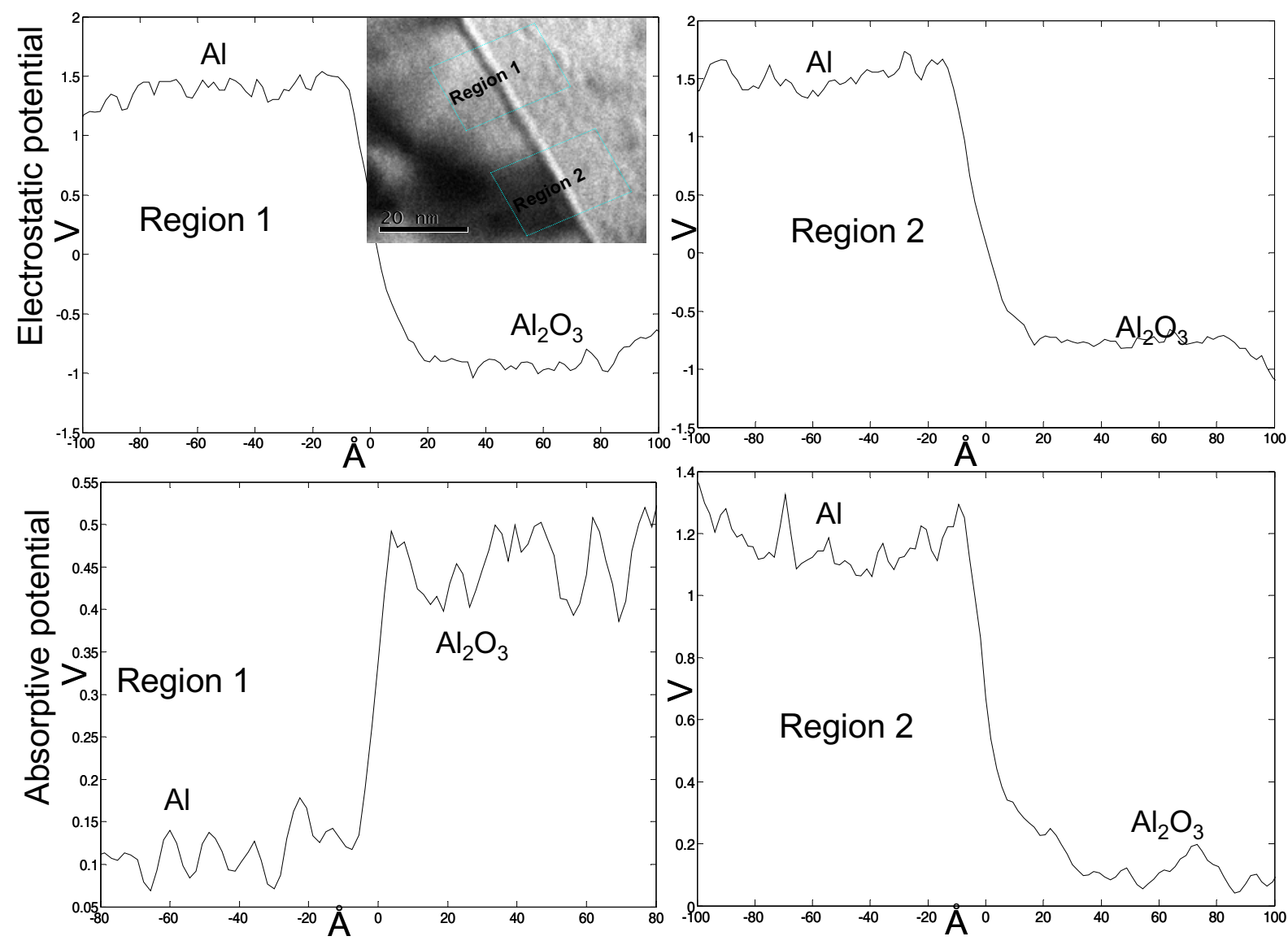

Fig. 2. Comparison of the reconstructed absorptive as well as the electrostatic potentials of an $\mathrm{Al} / \mathrm{Al} 2 \mathrm{O} 3$ interface. The line scans are taken from regions 1 and 2 of the image shown in the inset (overfocus of $2 \mu \mathrm{m}$, contrast variations due to local bending). 